\title{
Papers
}

\section{The publishing [R]evolution}

\section{Darin McBeath}

is Director of Disruptive Technology, Elsevier, responsible for researching new and emerging technologies and defining technical strategy. He represented Elsevier on the W3C XML Query Full Text Working Group and on the Medbiquitous Consortium Technical Steering Committee. For the past six years, he has specifically focused on XQuery and the opportunities this technology will offer publishers. He holds a Bachelor of Science degree in Systems Analysis from Miami University.

Keywords: $X M L, X Q u e r y, X M L$ database, publishing

Abstract Throughout history, the field of publishing has been profoundly influenced by technological advances ranging from Johannes Gutenberg's printing press to the creation of the World Wide Web by Tim Berners-Lee. Along the way, the term publisher has also been redefined to not only imply traditional publishers, such as Elsevier, but to additionally include governments, corporations and daily users of the internet. During the past decade, publishers have embraced the presentation neutrality of XML and the translation capabilities of XSLT to personalize a user's experience. But until now, the technology has not been available for a publisher to truly exploit database functionality for their XML content, repurpose these assets and unleash the power of XML. With the W3C XQuery 1.0 Recommendation in January 2007 and the introduction of XML Databases, the next significant technical advancement in publishing is currently underway and will forever change how XML assets are viewed, used and repurposed within the publishing industry. This paper provides some background information on these new technologies, describes how some publishers are unlocking their XML content with XQuery and XML Database technologies and presents some best practices identified through experience. Journal of Digital Asset Management (2007) 3, 231-238. doi:10.1057/palgrave.dam.3650100

\section{INTRODUCTION}

During the past decade, XML has been widely adopted by publishers as the preferred markup language for their structured assets. But many projects never went beyond leveraging this XML for primitive content repurposing to different device types or truly delivered on the promise and expense of migrating to XML. For many publishers, XML was the end game rather than the starting point. XQuery and XML Databases, however, are new technologies poised to change how digital assets stored as XML can be reused and further heighten the importance of not only solid structural markup within content but also semantic markup. These new tools will allow managers of digital assets to finally unlock their XML content to discover and repurpose assets in ways that were originally viewed as impractical or impossible plus generate new value by integrating XML content from different sources.
There are many parallels between the music industry and the publishing industry. The music industry has been revolutionized by the introduction of new content distribution channels. While legacy distribution channels were focused on complete CDs, these new distribution channels include individual songs and ring tones. The key point to remember is that the content for these new distribution channels is simply repackaged content from the original version. Similar changes are already occurring in the publishing sector. No longer is the distribution channel only a book. New distribution channels include chapters or learning bursts of information. Similar to the music industry, the content for these new distribution channels will be repackaged content from the original source. To enable this transformation in the publishing industry, XQuery and XML Databases will play a pivotal role. 


\section{WHAT IS XQUERY?}

The World Wide Web Consortium (W3C) XML Query (XQuery) Working Group ${ }^{1}$ has been working on the definition of XQuery, a language to process XML, since 1999. Leveraging other W3C Recommendations such as W3C XML Schema $1.0^{2}$ and W3C XQuery 1.0 XPath 2.0 Functions and Operators, ${ }^{3}$ this cross-industry group of XML experts published the W3C XQuery 1.0 Recommendation ${ }^{4}$ in January 2007. Additional W3C working groups are currently drafting recommendations to extend the original XQuery recommendation to include update ${ }^{5}$ and full-text search ${ }^{6}$ features.

But what is XQuery? The term XQuery is often referenced in a variety of contexts leading to confusion among vendors, developers and managers. Since it is difficult to craft a single and concise definition for XQuery, a particular view is often influenced by the XQuery implementation that has been adopted and what features of the language will be leveraged. The following are some common perceptions of XQuery.

Query language: Similar to SQL, XQuery can be used to execute a query against an underlying XML repository of content and return results. Transformation language: Similar to XSLT, XQuery can be used to transform content from XML to another format.

Integration language: XQuery can be used as the client-facing common query language for interacting with multiple disparate repositories. Programming language: By leveraging vendorspecific extensions, with some XQuery implementations it is possible to develop an application written completely in XQuery and not rely on other languages such as Java or $\mathrm{C} \#$.

Full-text search language: By leveraging vendorspecific extensions, with some XQuery implementations it is possible to search and retrieve content from an underlying XML repository.

None of the perceptions listed above are misperceptions of the XQuery language. While the views are very different, they are all correct given the specific context where XQuery is leveraged.

\section{XQUERY IMPLEMENTATIONS}

While there are currently more than 50 open source and commercial XQuery implementations listed on the W3C XQuery website, they can be categorized into one of the following general XQuery implementation types.

XQuery engine: These implementations provide the core capabilities outlined in the XQuery language, but provide no ability to persistently store $\mathrm{XML}$ documents. As a general rule, these implementations typically evaluate an XQuery request for one XML document at a time. Some vendors have added proprietary extensions, such as HTTP Get/Post, making XQuery a good solution for dynamic Web 2.0 mashups. For those engines that have not added these extensions, the XQuery engine can typically be combined with a web application to produce a powerful Web 2.0 framework. ${ }^{7}$

$X Q u e r y$ engine and XML database: In addition to the capabilities mentioned above, these implementations provide the ability to persistently store XML data. Because of the ability to persistently store XML data, these implementations usually provide the ability to evaluate an XQuery request over many XML documents stored in the underlying repository. Some implementations are from well-known RDBMS vendors while others are from new entrants specializing in the storage and retrieval of XML content.

Within the publishing industry, there tends to be a strong preference for not just an XQuery Engine implementation, but an XQuery Engine and XML Database.

\section{WHY XQUERY AND XML DATABASES?}

While there are many advantages for XQuery and XML Databases, the following have been identified through personal experience from building XQuery applications over the past six years. A common theme is the "perfect fit" that XQuery and XML Databases provide when developing solutions involving storing and retrieving XML content from a persistent repository. No longer must a developer try to fit the "square peg" of XML content into a "round hole" of previously attempted solutions. 
Match for document-centric applications: For many applications today, both the inputs and the outputs from a repository are often described in XML. Considerable time, however, is often spent parsing the inbound XML and breaking the content artificially into pieces so that the content can be managed and queried by a proprietary storage/retrieval system or RDBMS. When the content is retrieved from the underlying repository, this process must be reversed so that the original XML content is reconstituted. With XQuery and XML Database implementations, these intermediate transformations are hidden or no longer necessary depending on the particular implementation. This makes applications simpler to develop and maintain.

Speed of development and agility: This is really just an extension of the prior concept that reflects minimizing the number of transformations and code that must remain synchronized with the underlying stored XML content. For many applications, there are frequent changes in the XML markup contained in assets. But as the XML continually evolves throughout a project, fewer pieces need to be updated and coordinated if the application is developed using XQuery and XML Databases.

No predefined granularity: By leveraging XQuery with vendor provided full-text search extensions, products can easily be built with run-time defined search and retrieval granularity. With a traditional full-text search engine, search and retrieval granularity must be defined in advance. For example, if the granularity is a book chapter, the content must be indexed with this level of granularity. If the granularity is section, then the content needs to reindexed with this level of granularity. But, with XQuery and XML Databases, the XML content can be stored (indexed) once and products can be quickly built to search and retrieve entire documents or only parts of a document. This prospect is intriguing in today's world where a user is not searching for a complete document, but often for a fragment of information contained in a document.

Simplicity: Typically, a developer must create both a data model and a process model to solve a business problem. If there is already a schema for an XML document (or one can be easily created), why should it be necessary to create an
RDBMS schema? With XQuery and XML Databases, the developer can craft a solution based completely around the XML documents. While this simplifies the process, it requires a fundamental change in how problems are approached and requires thinking about XML very early in the process.

\section{WHY DO PUBLISHERS LIKE XQUERY AND XML DATABASES?}

Based on the observations listed above, it should be no surprise that the publishing sector is one of the largest adopters of XQuery and XML Databases. For example, a majority of the attendees at a recent XQuery user conference were from the publishing sector. The following responses were obtained from an informal survey of these publishing colleagues, when asked the question "Is XQuery a tool that enables publishers to unleash the power of XML and revolutionize the publishing industry?"

"I would agree with this, given the word 'enables'. We have only scratched the surface of how we can use XQuery (and XML databases) to provide unique capabilities with our content that were never possible before."

"What can it do that XSLT cannot? In combination with XML databases, XQuery is of course very powerful."

"XQuery brings a whole range of new capabilities and for the first time really enables publishers to 'database' their content and make effective use of it."

"We're able to search, mix data, and visualize in a way we've never been able to before. It's definitely going to change the way we think about content."

For years, publishers have been searching for a technology that will truly enable them to store, repurpose and reuse XML content. Now that this technology has arrived, how are publishers currently leveraging this new technology in their projects?

\section{XOUERY AND XML DATABASE USE CASES FOR PUBLISHING}

Below is a representative sample for how publishers are currently leveraging XQuery and XML Database technology within the enterprise. 
The particular applications range from small $100 \mathrm{MB}$ systems to $100 \mathrm{~TB}$ systems, single-tier architectures to multi-tier architectures and highly interactive real-time customer-facing retrieval systems to back-end electronic warehouse repositories. While the list is diverse, publishers are only beginning to scratch the surface for how XQuery and XML Databases can be leveraged within the enterprise.

Transformation: Instead of a Java/XSLT and proprietary storage or RDBMS solution, some publishers are leveraging XQuery and XML Databases to perform transformations on XML content loaded into a repository. In this model, the original content is loaded into the XML Database, transformed using XQuery and the output is stored back into the XML Database. Dynamic publishing: This could also be called the iTunes solution for publishing. In this model, entire books defined in XML are loaded into an XML Database and XQuery is used to construct "new" books from chapters of selected books stored in the XML Database. This allows users to create a personalized custom-constructed book.

Content analytics: This approach is frequently used to predict "what if" scenarios based on a publisher's XML content. For example, XQuery and XML Databases are a perfect solution to answer the question "How many caption elements exists on average for each document stored in a XML Database and what is the average size of the text contained in the caption?"

Search: Once the XML content is stored in an XML Database, some publishers are producing products to search the repository with different levels of granularity. For example, if a book is stored in an XML Database, vendor-provided XQuery full-text search extensions can be used to search and retrieve results for chapters, sections or paragraphs. The key point to remember is that the content must only be loaded (and indexed) once. Content repurposing: Often, when XML documents are loaded into an XML Database, new product ideas are frequently revealed because the original view of the asset was the document and not the internal structure. For example, it becomes easy for publishers to create new products for searchable image captions or searchable code samples. But this highlights the need for not only solid structural markup but also meaningful semantic markup within XML assets.

\section{XOUERY IMPLEMENTATION CONSIDERATIONS}

If these use cases highlight compelling opportunities within an enterprise, the next step is selecting a suitable XQuery implementation. The following partial list provides some representative criteria that should be considered when selecting an XQuery implementation. Like any other technology selection, factors such as price, support, scalability, reliability, training, etc should also be considered. The unique characteristics of a project could also identify additional criteria not included below.

Vendor features and extensions: Evaluation of vendor features and extensions is likely the most important consideration. A determination of what features and extensions are necessary will be dependent on the application being built. The following are the key considerations when conducting this evaluation.

- Do the project requirements necessitate an XQuery Engine or an XQuery Engine and XML Database?

- Does the vendor provide full-text search and database capabilities?

- Does the vendor support the final syntax defined in the W3C XQuery Recommendation or a prior syntax defined in a previous working draft?

- Does the vendor require a W3C XML Schema when loading content?

- What optional aspects of the W3C XQuery Recommendation are implemented by the vendor?

- If the vendor provides database capabilities, are transactions supported?

- Does the vendor provide the capability to develop applications completely written in XQuery?

- What indexing options are provided by the vendor to improve performance?

Performance characteristics: Another important criterion will be the performance characteristics of the implementation for the application's $\mathrm{XML}$ content and respective queries. Within the XQuery community, there are currently no broadly endorsed 
performance benchmarks such as the TPC $^{8}$ benchmarks. For example, some implementations will perform well on small XML document retrievals while others will perform well on larger XML document retrievals. Similarly, some implementations can quickly perform node-level updates while others excel with entire document updates. Careful consideration should be given to the target XML content and how it will be queried and updated by the implementation.

Prototype evaluation: During the early adoption lifecycle of any new technology, it is extremely important to construct working prototypes for evaluating the technology and a particular vendor's implementation of the technology. While a certain amount of evaluation and comparison can be performed from reading vendor-provided documentation, it is truly important to construct and evaluate a prototype to get a first-hand feel for the technology and minimize the number of unexpected surprises when developing a real application.

Skillset considerations: Since XQuery is a fairly new language, it will be difficult to find plentiful developers seasoned with experience. Fortunately, XQuery leverages many concepts found in some currently well-known and widely adopted technologies. Enterprises wanting to incorporate XQuery into a project will want to find developers with comparable experience in several of the following technologies.

- XML (W3C Schema)

- XPath

- XSLT

- Functional programming (such as Scheme or Lisp).

\section{XQUERY INTEROPERABILITY CONSIDERATIONS}

As mentioned previously, all XQuery

implementations are not created equal. For each XQuery implementation, there is a range of functionality and features provided by the vendor. A common source of differences in the implementations is a result of vendor-provided extensions to the XQuery language or vendor interpretations for loosely or not yet defined aspects of the XQuery language specification. Another source of differences is the W3C XQuery 1.0 Conformance ${ }^{9}$ section, which allows parts of the W3C XQuery 1.0 Recommendation to be "optional"; yet the implementation can still be considered XQuery 1.0 compliant. Since the W3C XQuery 1.0 Recommendation is fairly recent, there are also syntactical language differences between implementations due to earlier versions of the specification being implemented by some vendors.

For the next several years, the task of porting XQuery modules developed on one implementation to another implementation will not be a trivial task. If XQuery modules are developed using a basic XQuery Engine, the task should be fairly straightforward. This could be hampered, however, by the conformance concept mentioned previously or if vendor extensions are leveraged.

For XQuery modules that leverage an underlying database to persistently store XML content, this will be a more complex task. By default, the concept of simply loading (or updating) a document into an XML Database requires a vendor extension since there is currently no update standard for XQuery. While there are $\mathrm{W} 3 \mathrm{C}$ working groups addressing $\mathrm{XQuery} \mathrm{language} \mathrm{missing} \mathrm{features} \mathrm{such} \mathrm{as}$ update and full-text search, it will be some time before these standards are published and adopted by implementations. It is also too early to discern whether these additional recommendations will completely deliver all of the necessary functionality to replace the current vendor proprietary extensions.

When invoking XQuery modules from higher level languages such as Java, the client code currently requires proprietary APIs offered by the vendor. The longer term solution will likely be XQJ/JSR $255,{ }^{10}$ a JDBC-type API for XQuery designed and supported by many of the organizations participating in the development of XQuery. But, once again, there will be a time lapse before the XQJ API is officially published and widely adopted by XQuery implementations. Unfortunately, if the client language is something other than Java, the XQJ API will be of little help since it will only provide support for the Java programming language.

Nonetheless, it is obvious there will be vendor "lock-in" for the next few years. To minimize the risk, it is important that 
the features provided by the vendors and the requirements of the project are well understood before selecting an XQuery implementation.

\section{XOUERY BEST PRACTICES}

Before incorporating XQuery into an application, an organization should identify and adopt some consistent approaches and guidelines for utilizing this technology. With the current differences in XQuery engines available in the market, many best practices are tailored for a specific implementation. These best practices are often communicated and discovered from participating in implementation-specific discussion groups. Nonetheless, it is still possible to identify some general XQuery best practices for style, documentation, design and testing.

\section{Style considerations}

Style conventions are important for a number of reasons:

- Common style conventions will decrease the longterm maintenance costs associated with XQuery modules.

- Hardly any XQuery module will be maintained for its whole life by the original author.

- Style conventions improve the readability of the XQuery modules, allowing everyone to understand the XQuery more quickly and thoroughly.

XQuery style conventions will not ensure that the XQuery authored for one XQuery engine will work on a different XQuery engine. If the migration to a different XQuery engine becomes necessary, however, this task can be simplified by adhering to a consistent style when authoring XQuery modules.

The XQuery Style Conventions ${ }^{11}$ are a good starting point for some vendor-neutral style conventions. Similar to the Java coding conventions, this document offers ideas for module and code organization to thoughts on variable and function-naming conventions. Many of the suggestions contained in the document were validated based on input and feedback from the XQuery development community.

\section{Documentation considerations}

When developing any software, it is important to minimize the effort required to keep software documentation current. The XQuery language is no different from any other programming language. Similar to the features offered by Javadoc for the Java programming language, $\mathrm{xqDoc}^{12}$ provides a simple vendor-neutral solution for documenting XQuery modules, as well as tools to generate a user-friendly presentation of this documentation and crossreference information. The xqDoc tool was initially developed by Elsevier to assist their development team with understanding the growing complexity of their XQuery applications and minimizing the XQuery learning curve for new team members. Since that time, the tool has been released to the open-source community and incorporated into two commercial XQuery IDEs.

\section{Design considerations}

In practice, many current design considerations tend to be vendor specific and not necessarily applicable across all XQuery implementations. But it is possible to identify some key vendorneutral design guidelines. The following, abstracted from the previously mentioned XQuery Style Conventions, are a sampling of these vendor-neutral design considerations. As XQuery development continues to mature and lessons are learned, additional design best practices will be identified.

Main modules: Main modules should be viewed as the "public" interface for the XQuery application. Typically, functions and variables should not be declared in a main module since this prevents any other main module or library module from using these variables or functions. The exception to this rule would be if the intent is to minimize the scope of these functions and variables by essentially making them hidden or private.

Library modules: Library modules should be viewed as the "private" interface for the XQuery application. All functions and variables should be declared in a library module since this allows any other main module or library module to reuse these variables or functions. It is also a best practice to group functions and variables used for a common purpose into the same library module. 


\section{Vendor extensions}

Vendor-provided extensions to the XQuery language should be avoided unless they add significant value. While this is currently unavoidable for applications leveraging proprietary full-text search and update features provided by vendors, each extension should be closely scrutinized since this will further couple the application to a particular XQuery implementation. A future migration to a different XQuery implementation will be complicated with the usage of each proprietary vendor extension.

\section{Testing considerations}

While the concept of unit testing is nothing new to the software industry, the necessity of unit testing for an XQuery application should not be ignored. With XQuery's premise as a function-based programming language, the importance of testing the individual building blocks of functions should be obvious. Unfortunately, this is not always the case, and issues that could have been identified within minutes take days of debugging.

There are currently no widely adopted frameworks, such as JUnit, for unit testing XQuery functions. Those that have embraced the importance of unit testing have developed homegrown frameworks by extending JUnit or XMLUnit. Some popular XQuery IDEs have also started to provide traditional debugging capabilities to assist with development of quality XQuery code. While these basic advancements of providing break points and watch variables are a huge step forward, the features are typically only available for a handful of XQuery implementations.

\section{XOUERY POPULARITY}

With any new technology, there is often a period of hype and exaggeration by the vendors, with only limited uptake or adoption in the software development community. While XQuery is no exception, the past several years have witnessed increases in the number of conference presentations (Figure 1), research papers (Figure 2) and conference attendees involving XQuery (Figure 3).

\section{CONCLUSION}

While the XQuery and XML Database

landscape is still evolving and maturing, many

\begin{tabular}{|c|c|}
\hline Year & Number of XQuery Presentations \\
\hline 2005 & 3 \\
\hline 2006 & 9 \\
\hline
\end{tabular}

Figure 1: Number of "XQuery" presentations for the past couple of years at the annual XML Conference sponsored by IDEAlliance ${ }^{13,14}$

\begin{tabular}{|c|c|}
\hline Year & Number of XQuery Published Papers \\
\hline 2003 & 38 \\
\hline 2004 & 58 \\
\hline 2005 & 112 \\
\hline 2006 & 119 \\
\hline
\end{tabular}

Figure 2: Number of published papers for the past several years with "XQuery" in the title, abstract or keywords. Data obtained from Elsevier's Scopus ${ }^{15}$ research tool

\begin{tabular}{|c|c|}
\hline Year & Number of Conference Attendees \\
\hline 2004 & 22 \\
\hline 2005 & 53 \\
\hline 2006 & 100 \\
\hline 2007 & 236 \\
\hline
\end{tabular}

Figure 3: Number of attendees for the past several years at one commercial XQuery and XML Database vendor's user conference

publishers have already built production applications leveraging this new technology. More are building prototypes to further evaluate the possibilities of leveraging XQuery and XML Databases for projects within the enterprise. While there might have been difficulty with justifying an expensive and long migration to XML in the past, these new technologies offer exciting opportunities for opening the XML content vault and justifying the business case. No longer will XML assets be viewed as documents; instead, the document's internal structure of paragraphs and sentences will be viewed as building blocks of information that can be repurposed and reused to manufacture new and exciting products.

Common themes throughout the publishing industry today are speed and flexibility. Publishers not only want the flexibility to create new products but additionally bring these products to market quickly. To achieve these goals, several publishers are leveraging XQuery and XML Databases and are witnessing the following measurable benefits when contrasted with legacy approaches. 
- Improved content loading times.

- Reduction in prototype development cycles.

- Reduction in overall development schedules.

- Improved ability to slice/dice content to create new products.

As these success stories become further disseminated, there is little doubt that XQuery and XML Databases will play a pivotal role in the future of digital asset management systems for structured content and a key enabler for the Publishing [R]evolution.

\section{References}

1 W3C World Wide Web Consortium, XML Query Working Group, http://www.w3.org/XML/Query/.

2 W3C World Wide Web Consortium, XML Schema Datatypes, http://www.w3.org/TR/xmlschema-2/.

3 W3C World Wide Web Consortium, XQuery 1.0 and XPath 2.0 Functions and Operators, W3C Recommendation, http://www.w3.org/TR/xpath-functions.

4 W3C World Wide Web Consortium, XQuery 1.0: An XML Query Language, W3C Recommendation, http://www.w3.org/TR/Query.
5 W3C World Wide Web Consortium, XQuery Update Facility 1.0, W3C Working Draft, http:// www.w3.org/TR/xquery-update-10/.

6 W3C World Wide Web Consortium, XQuery 1.0 and XPath 2.0 Full-Text 1.0, W3C Working Draft, http://www.w3.org/TR/xquery-full-text/.

7 Yan, N. Power your mashups with XQuery http:// www.ibm.com/developerworks/xml/library/ x-xquerymashup/index.html.

8 Transaction Processing Performance Council, TPC Benchmarks, http://www.tpc.org/.

9 W3C World Wide Web Consortium, XQuery 1.0: An XML Query Language - Section 55 Conformance, W3C Recommendation, http://www.w3. org/TR/xquery/\#id-xquery-conformance.

10 Java Community Process, JSR 225: XQuery API for Java, http://jcp.org/en/jsr/detail?id=225.

11 McBeath, D. XQuery style conventions http:// xqdoc.org/xquery-style.pdf.

12 McBeath, D., xqDoc - XQuery documentation project, http://www.xqdoc.org.

13 IDEAlliance, XML 2005 Conference Program, http://www.idealliance.org/proceedings/xml05/ indices/titles.html.

14 IDEAlliance, XML 2006 Conference Program, http://2006.xmlconference.org/programme/.

15 Elsevier, Scopus, http://www.scopus.com. 